University of Wollongong

Research Online

Faculty of Engineering and Information

Faculty of Engineering and Information

Sciences - Papers: Part B

Sciences

2018

\title{
Applying Mechanical Pressure and Skin Stretch Simultaneously for Sensory Feedback in Prosthetic Hands
}

\section{Benjamin Stephens-Fripp}

University of Wollongong, bsf147@uowmail.edu.au

Rahim Mutlu

University of Wollongong, rmutlu@uow.edu.au

Gursel Alici

University of Wollongong, gursel@uow.edu.au

Follow this and additional works at: https://ro.uow.edu.au/eispapers1

Part of the Engineering Commons, and the Science and Technology Studies Commons

Research Online is the open access institutional repository for the University of Wollongong. For further information contact the UOW Library: research-pubs@uow.edu.au 


\title{
Applying Mechanical Pressure and Skin Stretch Simultaneously for Sensory Feedback in Prosthetic Hands
}

\author{
Abstract \\ An effective method of communicating sensory feedback for prosthetics is presented using a \\ combination of mechanical pressure and skin stretch, resulting in a mixture of normal and shear force \\ being applied to the human arm. Stimulations were induced on the subject's forearm by three mechanical \\ cranks, each attached to their own servo motor. Three different crank orientations were tested, each \\ producing a different skin stretch direction, with the results showing that shear force/tangential skin \\ stretch applied longitudinally to the forearm was perceived more easily as it produced the best \\ recognition rate. With minimal training, eighteen able-bodied test subjects were able to recognise six \\ different grips with an accuracy of up to $88 \%$, and achieved an accuracy of $80 \%$ when recognising the six \\ grips at two different pressure levels. This sensory feedback mechanism shows potential for a simple, \\ easy to learn stimulation device that could help improve users control and embodiment of their prosthetic \\ device that requires three separate feedback channels.

\section{Disciplines} \\ Engineering | Science and Technology Studies

\section{Publication Details} \\ Stephens-Fripp, B., Mutlu, R. \& Alici, G. (2018). Applying Mechanical Pressure and Skin Stretch \\ Simultaneously for Sensory Feedback in Prosthetic Hands. 2018 7th IEEE International Conference on \\ Biomedical Robotics and Biomechatronics (Biorob) (pp. 230-235). United States: IEEE.
}




\title{
Applying Mechanical Pressure and Skin Stretch Simultaneously for Sensory Feedback in Prosthetic Hands
}

\author{
Benjamin Stephens-Fripp, Rahim Mutlu, Gursel Alici
}

\begin{abstract}
An effective method of communicating sensory feedback for prosthetics is presented using a combination of mechanical pressure and skin stretch, resulting in a mixture of normal and shear force being applied to the human arm. Stimulations were induced on the subject's forearm by three mechanical cranks, each attached to their own servo motor. Three different crank orientations were tested, each producing a different skin stretch direction, with the results showing that shear force/tangential skin stretch applied longitudinally to the forearm was perceived more easily as it produced the best recognition rate. With minimal training, eighteen able-bodied test subjects were able to recognise six different grips with an accuracy of up to $88 \%$, and achieved an accuracy of $80 \%$ when recognising the six grips at two different pressure levels. This sensory feedback mechanism shows potential for a simple, easy to learn stimulation device that could help improve users control and embodiment of their prosthetic device that requires three separate feedback channels.
\end{abstract}

\section{INTRODUCTION}

Tactile information is required to plan and control object grasps and manipulations as vision alone does not provide enough of the information required [1]. Prosthetic users have also shown a strong desire to decrease the need for visual attention to perform functions [2]. Prosthetic hand rejection rates are estimated to be as high as $40 \%$ [3], with some of the user's reasons to reject or simply not wear their prosthetic include that they believe it is more functional and easier to receive sensory feedback through their stump without using the prosthetic hand [4]. Sensory feedback is also important for prosthetics as it can also provide users with a sense of embodiment in their prosthesis [5-7].

Grasping force feedback is the highest priority for sensory feedback of prosthetic users, followed by sensing the position of their prosthetic digits [8]. Ninu et al. [9] and Schweisfurth et al. [10] suggest that initial force grasping of objects can be achieved by predicting initial grasping force. One method is using velocity of closing grasp to provide information for initial force.

There are a number of tactile sensing methods found within the literature [11]. Mechanotactile information can be easier to discriminate than vibrotactile information [12]. Wearable haptic devices have had some previous success in sensory

B. Stephens-Fripp, G. Alici and R. Mutlu are with the Intelligent Nano-Tera Systems Research Laboratory, based at the School of Mechanical, Materials, Mechatronic and Biomedical Engineering, University of Wollongong, Australia; and are part of the ARC Centre of Excellence for Electromaterials Science, University of Wollongong, Australia. (Corresponding author: Gursel Alici - gursel@uow.edu.au). feedback with winding belts being used to feedback information on grasping force [13, 14], and the hardness of the object [15], through changing pressure and skin stretch on the bicep. However, these methods only provided one channel feedback to the user and were bulky. Similarly, a rocker design has been used to communicate proprioceptive information through skin stretch [16], however, it also only communicates one degree of actuation.

Linear skin stretch has also been used to communicate force feedback [17] though pulling silicon bulbs attached to the user's forearm; and in grip recognition [18], where the motor also pulls a contact pad attached to the forearm. Although both produced encouraging accuracy, they added large bulk to the prosthesis due to the nature of the driving system, and the skin attachment methods were impractical in the attachment for daily use and can be easily detached after several uses.

The use of five servo controlled mechanical pressure devices was demonstrated by Antfolk et al. [19] for grip recognition and force feedback. These vertical pressure devices, however, only achieved a grip recognition accuracy of $58 \%$ for amputees and $68 \%$ for able-bodied participants, with a high amount of confusion occurring between neighbouring areas.

In this study, we propose an improved method of mechanotactile feedback, to that used by Antfolk, by using three servo controlled mechanical cranks which combine vertical pressure with linear skin stretch when providing sensory feedback. The number of feedback channels were limited to three; to represent the movement of the thumb, the pointer finger and the remaining three fingers. Antfolk et al. [20] reported an average discrimination rate of $97 \%$ for three feedback channels using mechanotactile devices, compared to an average discrimination rate of $82 \%$ for 5DOF. Prosthetic hands with three degrees of freedom are one common approach taken $[17,18,21]$. The grasping taxonomy used by Vergara et al. [22] to record the frequency usage of different grasps also does not require independent movement of the ring and little fingers.

Pilot testing of this experiment demonstrated that individual users had different comfort tolerance with the mechanical cranks, and differences existed between the comfort levels across the three stimulation sites and different orientations. Therefore, to increase the comfort level for the test subjects and to help increase perception recognition, all three mechanical crank stimulation sites were calibrated separately for each individual user and for each orientation tested. 
This experiment was conducted in two parts. In Part A, the time taken to reach maximum displacement was measured since previously published literature has shown that a delay of greater than $300 \mathrm{~ms}$ can decrease embodiment with sensory feedback [23, 24]. Part B measured and compared the recognition rates of subjects with the three different orientations of the mechanical cranks; transversally, longitudinally and diagonally to the arm as demonstrated in Figure 4; to determine which direction the shear stress/translational skin stretch is more easily perceived on the human forearm.

\section{METHOD}

The proposed mechanical crank feedback system is shown in Figure 1. It consists of three Goteck micro servo-motors, controlled via a microcontroller with a LabVIEW Interface. The mechanical cranks were custom $3 \mathrm{D}$ printed to match the length of the motor, with a depth of $5 \mathrm{~mm}$. A surfboard leash cuff (Smart Leash Co.) was used to hold them firmly against the user's skin. The servos were mounted to a 3D-printed frame, which was then attached to the cuff.

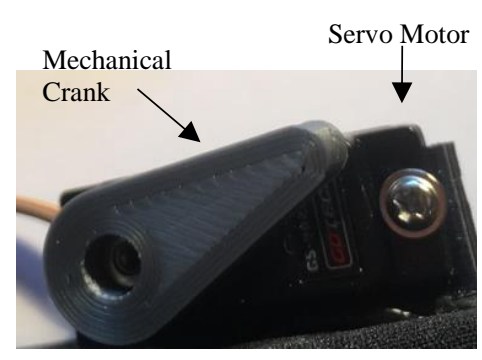

(a)

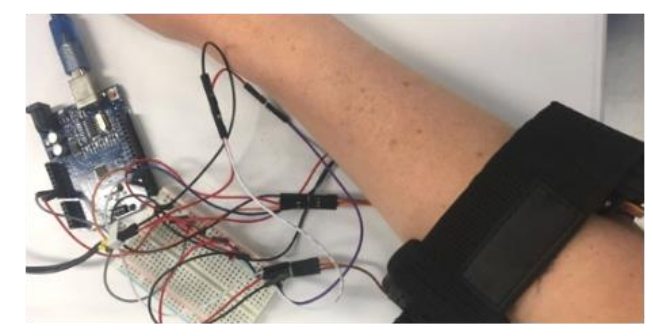

(c)

Figure 1 - Mechanical Crank Feedback

(a) Mechanical Crank , (b) Crank location on cross-section of arm and, (c) Placement on arm

\section{A. Part A}

To measure the time taken to begin activation of the feedback mechanism, as well as the time to complete the movement, a mechanical crank attached to a servo motor was fixed into place and its movements detected through a measurement laser (Micro-Epsilon). The laser 1 detected the initial movement time when the trailing edge began moving, as shown in Figure 2a; and the finished movement was measured from the detection of the leading edge reaching the maximum displacement detected by laser 2, shown in Figure 2b. A LabVIEW interface was used to control the servo motor, via a microcontroller, and operate the millisecond precision timer. A flowchart of its process is shown in Figure 3, which was repeated ten times. An average time of $53.4 \mathrm{~ms} \pm 9.5 \mathrm{~ms}$ (S.D.) was recorded for the servo to begin movement. This time consists of the time taken for the microcontroller to process and send the command (measured at 22ms), as well as start-up time of the motor to drive dynamics and stiction. An average time of $162.4 \mathrm{~ms} \pm 6.6 \mathrm{~ms}$ was recorded for the full servo movement from when the command was sent, which is lower than $300 \mathrm{~ms}$ proposed in the literature. However, this may slow down when the movement is pressing onto a human arm skin at the point of contact.

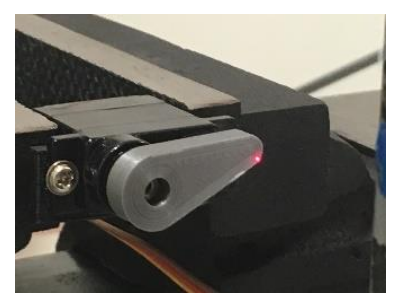

(a)

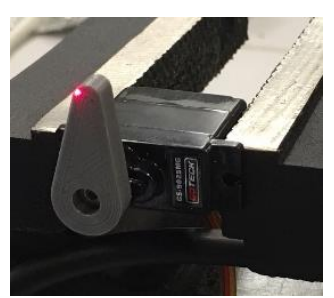

(b)
Figure 2 - Mechanical Crank Timing Experiment Setup; (a) Measuring starting movement from trailing edge, (b) Measuring finished movement by detecting leading edge

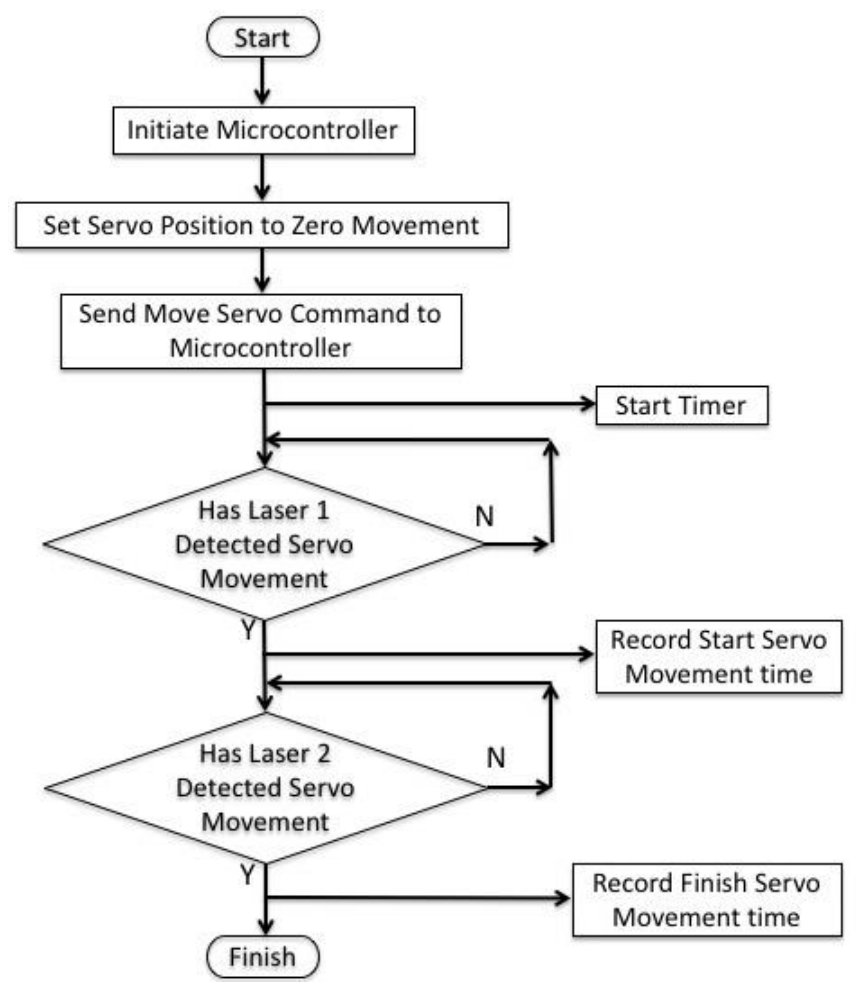

Figure 3 - Laser Timing Flowchart.

\section{B. Part B}

The range of movement of the crank for each user was determined through a calibration routine, where the system slowly increased the range of movement, resetting back to the zero position each time, to determine the largest crank movement comfortable. The user indicated when it was no 
longer comfortable, and the last comfortable movement was set as the maximum displacement for the user. This process was repeated for each crank.

Three orientations of crank movement to the forearm were compared: longitudinally, transversally and diagonally at an angle of 45 degrees, as shown in Figure 4. Performance was measured by the accuracy in recognition of grip patterns and intensity of pressure based on the amount crank rotation.

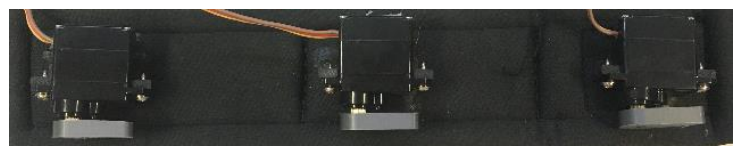

(a)

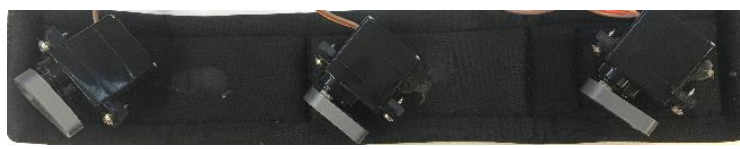

(b)

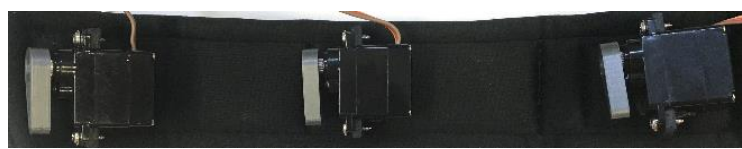

(c)

Figure 4 - Mechanical Crank Orientations; (a) Transversal, (b) Diagonal and, (c) Longitudinal

Recognition of six different grip patterns, shown in Figure 5, was tested: thumb only, pointer only, pistol grip (closing remaining three fingers only), fine grip (closing thumb and pointer), tool grip (closing thumb and remaining three fingers) and power grip (closing all fingers). These are commonly used grip patterns to test prosthetic feedback $[18,25]$. Each of these grips were tested in the fully closed position, represented by maximum comfortable crank displacement of the servo; or half-closed position, represented by $50 \%$ of the maximum comfortable angular displacement.

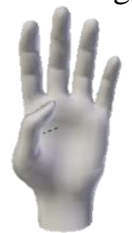

(a)

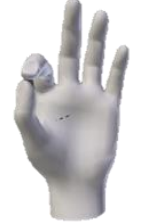

(d)

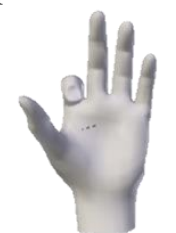

(b)

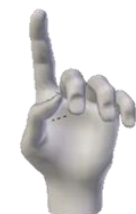

(e)

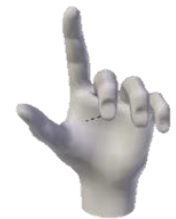

(c)

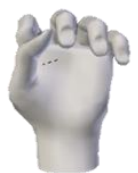

(f)
Figure 5 - Hand Grips: (a) Thumb Only, (b) Pointer only, (c) Pistol Grip, (d) Fine Grip, (e) Tool Grip and, (f) Power Grip

In the training phase, each of the six finger movements were demonstrated to the user at maximum displacement. The movement was communicated to the user prior to commencing sensory feedback, both verbally and visually with a picture of the corresponding grip. The crank stayed in maximum displacement for a period of $800 \mathrm{~ms}$ before returning to zero displacement, where there was a pause of five seconds before the next movement took place. After six movements, a 20second-long break occurred before repeating all the grips at $50 \%$ displacement. A two-minute break then occurred prior to the commencement of the testing phase. This short training period was used to demonstrate that due to intuitive nature of understanding the communicated feedback, extensive training is not required to achieve successful results.

In the testing phase, a randomised order of the six movements with three repetitions was developed, resulting in a total of 18 movements. Half of these movements were randomly assigned as maximum displacement and the other half were assigned $50 \%$ displacement. Each test subject had their own randomised movement and strength combinations, presented to them in their own randomised order. The grips were held at the displacement for $800 \mathrm{~ms}$ before returning to zero displacement. There was at least a five-second pause between each movement for the subject to communicate the perceived movement. The subject could verbally tell the grip perceived or could choose the grip picture in a chart corresponding to those shown in Figure 5. This process was repeated for the two other crank orientations, with a fiveminute break in between each orientation test. A total of 18 subjects was tested, consisting of 16 males and 2 females, with no physical or cognitive impairment. The order of the orientation tested was changed for each subject to prevent the effect of additional training influencing the results. In total, the six different combinations the testing orders were repeated three times across the 18 subjects. Statistical analysis was performed using SPSS (IBM SPSS V24, IBM Armonk NY). Written informed consent was obtained from all individuals participating in the study and ethical approval was obtained from the University of Wollongong Human Research Ethics Committee.

\section{RESULTS AND DISCUSSION}

\section{A. Grip Only}

The average recognition rates for the different orientations are shown in Table 1 and Figure 6. A repeated measures ANOVA with a Greenhouse-Geisser correction determined that the mean recognition performance different statistically significantly between orientations $(\mathrm{F}(1.552,26.387)=4.970, \mathrm{P}$ $=0.021)$. Post hoc tests using the Bonferroni correction revealed that longitudinal orientation $(88.0 \% \pm 6.9 \%)$ produced an increase in performance against transversal orientation $(78.4 \% \pm 10.4 \%)$ with a statistical significance of $\mathrm{P}=0.006$; and an improved recognition rate compared to diagonal orientation $(78.4 \% \pm 15.7 \%)$ with a statistical significance of $\mathrm{P}=0.035$. The difference in performance between transversal and diagonal orientation was not significant $(\mathrm{P}=1.000)$. A confusion matrix for grip recognition from all orientations combined and from the best performing orientation (longitudinal) are shown in Figures 7 and 8, respectively.

While normal and sheer pressures are induced in each crank orientation, shear stress/tangential skin stretch appears to be interpreted easier when applied longitudinally to the human arm as it results in the highest recognition rate. 
TABLE I. RECOGNITION RATE OF GRIP ONLY

\begin{tabular}{|c|c|}
\hline Orientation & Average \% Recognition \pm SD \\
\hline Longitudinal & $88.0 \% \pm 6.9 \%$ \\
\hline Transversal & $78.4 \% \pm 10.4 \%$ \\
\hline Diagonal & $78.4 \% \pm 15.7 \%$ \\
\hline
\end{tabular}

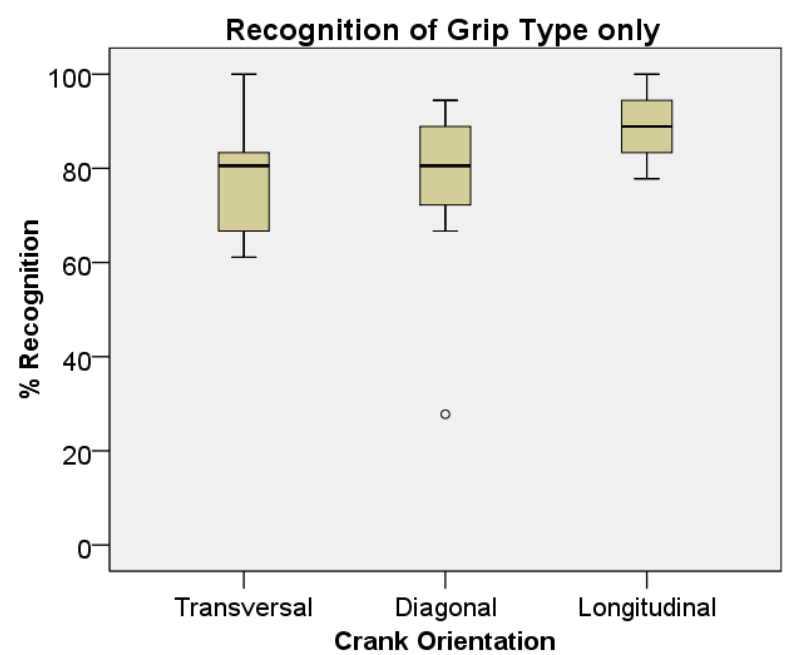

Figure 6 - Box Plot: Recognition Rate of Grip only; where: the dark line represents the median, The box indicates the Interquartile Range (IQR) and the whiskers represents either the $\mathrm{max} / \mathrm{min}$ or 1.5 times the IQR (whichever is closer); Circle represent value $>1.5 \times \mathrm{IQR}$

Figure 7 - Confusion Matrix for Grip from all orientations

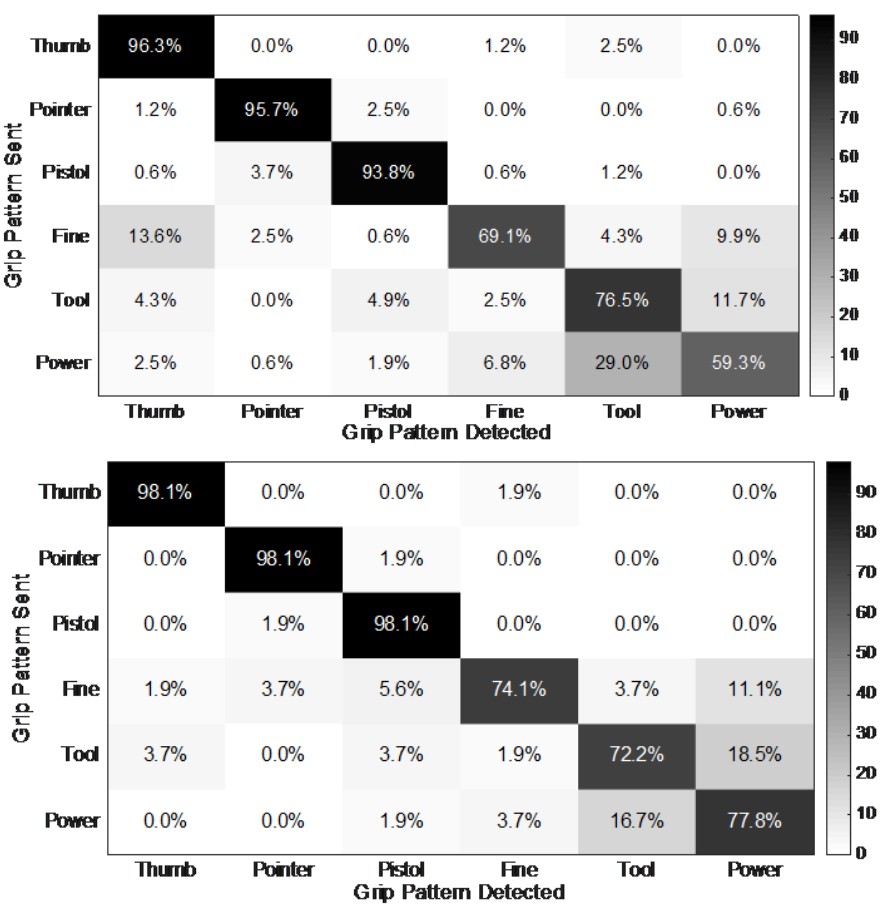

Figure 8 - Confusion matrix of Grip for Vertical Orientation

\section{B. Grip and Intensity Combined}

The average recognition rates for the different orientations are shown in Table 2 and Figure 9. A repeated measures ANOVA with a Greenhouse-Geisser correction determined that the mean recognition performance different statistically significantly between orientations $(\mathrm{F}(1.580,26.865)=7.284 \mathrm{P}$
$=0.005)$. Post hoc tests using the Bonferroni correction revealed that longitudinal orientation $(80.9 \% \pm 11.6 \%)$ produced an increase in performance against transversal orientation $(68.2 \% \pm 13.7 \%)$ with a statistical significance of $\mathrm{P}=0.009$; and an improved recognition rate compared to diagonal orientation $(69.8 \pm 16.3 \%)$ with a statistical significance of $\mathrm{P}=0.002$. The difference in performance between transversal and diagonal orientation was not significant $(\mathrm{P}=1.000)$.

\section{TABLE II. RECOGNITION RATE OF GRIP AND INTENSITY COMBINED}

\begin{tabular}{|c|c|}
\hline Orientation & Average \% Recognition \pm SD \\
\hline Longitudinal & $80.9 \% \pm 11.6 \%$ \\
\hline Transversal & $68.2 \% \pm 13.7 \%$ \\
\hline Diagonal & $69.8 \pm 16.3 \%$ \\
\hline
\end{tabular}

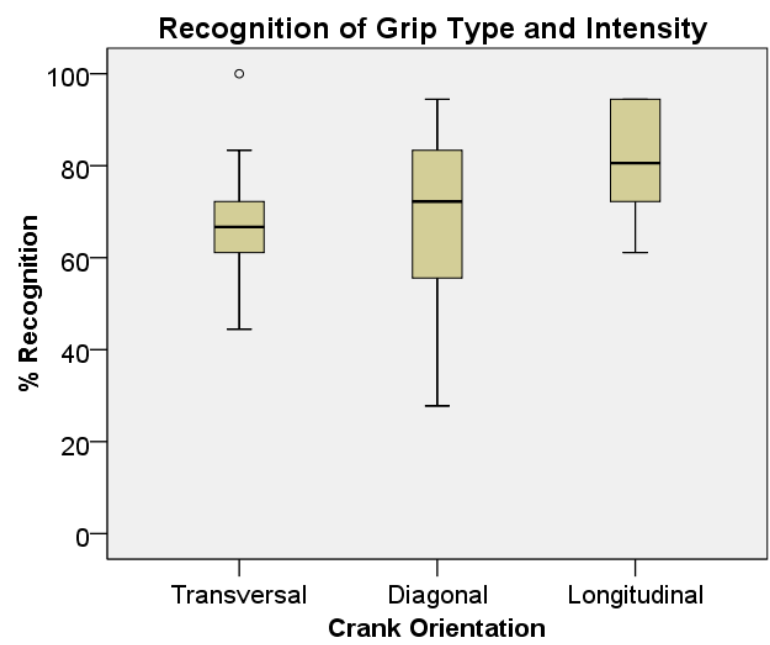

Figure 9 - Box Plot: Recognition Rate of Grip and Intensity Combined, where: the dark line represents the median, The box indicates the Interquartile Range (IQR) and the whiskers represents either the max/min or 1.5 times the IQR (whichever is closer); Circle represent value $>1.5 \times$ IQR

Considering the small training time, with only one demonstration of each grip at both force levels, subjects achieved a high recognition rate of both grip and force levels. The training also only incorporated visual pictures and verbal labels of grips. Although there were promising results with minimal training, increased learning time with a visualisation of a prosthetic hand moving, either real or virtual reality, could still help further increase the accuracy. Some testing subjects used their previous prediction to help determine what grip and/or intensity the next stimulation was, without knowing whether their previous prediction was correct, which sometimes resulted in multiple incorrect recognitions. In real world situations, however, subjects would incorporate visual feedback as a truth basis for continual learning to help improve their recognition rates.

An analysis was performed to determine if there was any significant impact on the order of testing, independently of the orientation they used. A repeated measures ANOVA with a Greenhouse-Geisser correction determined that the mean recognition performance that contained no statistically significant difference for the order of testing for Grip only $(\mathrm{F}(1.605,27.279)=1.728, \mathrm{P}=0.200)$. However, there was a 
small statistically significant difference between order of testing when examining grip and intensity combined $(\mathrm{F}(1.879,31.935)=3.927, \mathrm{P}=0.32)$. Post hoc tests using the Bonferroni correction revealed that the second trial $(77.5 \% \pm$ $12.8 \%$ ) produced an increase in performance against the first trial $(67.0 \% \pm 16.1 \%)$ with a statistical significance of only 0.042 , but no statistical difference compared to the third trial $(72.5 \% \pm 13.0 \%)$ with $\mathrm{p}=0.577$. The first and third trial showed also showed no significant difference $(p=0.447)$.

These results are an improvement upon the results reported by Antfolk et al. [19], who achieved an average accuracy of $68 \%$ for their able bodied participants. In their study, five out of ten of their participants were amputees, however, they noted that there was no statistical difference between able bodied subjects and amputees for the grip recognition and distinguished level of touch experiments. Our experimental evaluation tested recognition of a larger number of grip patterns, examining six grip patterns at two different force levels, totalling 12 different possible options; compared to Antfolk et al.'s testing of three different grips, with only one grip containing three different force levels, totalling five different grip options. Therefore, since our lowest result was comparable to the previously obtained results, whilst incorporating twice as many grip options, this result demonstrates the benefit of using the skin stretch action when applying pressure through the use of the mechanical crank. Further, our results indicate that this skin stretch is most effective when applied longitudinally to the human arm.

As shown in the confusion matrices (Figures 7 and 8), errors were made when multiple motors are activated at once (Fine, Tool and Power Grip). Currently the motors and cranks rest on the skin when no movement occurs. This may make it difficult to distinguish between when a crank is moving against your skin and when the motor/crank is pulled against you from movement of another crank. Adding a layer of padding underneath the motors, with gaps for the crank to go through, could improve the comfort level and help reduce false detections. Verbal feedback from the subjects was that the crank on the middle motor, corresponding to the pointer finger, was the hardest to detect when multiple motors were activated. Although individually calibrating each crank aimed to reduce any difference in perception between the motors, it could be further improved by operating the cranks using a constant force feedback method where an intensity is communicated by the crank supplying a corresponding force, rather than the currently utilised method of intensity corresponding to crank displacement.

Within this experiment, each person used the same armband with the same spacing, however, there were large variances in the size of the subject's arms. Further improvements could be made in comfort and recognition rate by using different armbands specific to the size of the subject's arm. In addition, further improvements could be achieved by each servo motor being attached to their own separate armband, so that when one motor activates it does not unintendedly pull another motor into the skin by stretching the armband.

\section{CONCLUSION}

This study demonstrated an effective and low cost mechanotactile approach that could be used in either grasping force feedback or position feedback for a prosthetic hand with three channels of feedback. With a short training period, recognition rates of up to $80 \%$ were achieved with six different grip patterns at two different intensity levels. This approach has the advantage of being easily applied, removed, adjustable location, only adds minimal bulk and has a maximum delay time of $162 \mathrm{~ms}$.

In achieving the similar results as Antfolk et al. [19] with more than twice as many grip options, this study has demonstrated the benefit of combining skin stretch with the vertical pressure. The skin stretch was also demonstrated to result in a better result when applied longitudinally to the forearm, shown by the statistically significant improvements in recognition rate compared to the other orientations.

Improvements can be made by removing the contact of the motors from the skin, and using force feedback control for the mechanical crank motors. Future work should also focus on the use of these mechanical cranks to provide real time feedback to determine if it improves the force control of a prosthetic arm. Although subjects recognised the type of grip and their force level statically with a reasonable level of accuracy, it has yet to be demonstrated whether this can be incorporated into the user's control feedback loop for dynamic real-time feedback. Testing was all done on ablebodied participants and follow up experimentation is required on amputee subjects.

\section{ACKNOWLEDGMENT}

This research has been conducted with the support of the Australian Government Research Training Program Scholarship, and with the support of the ARC Centre of Excellence for Electromaterials Science (Grant No. CE140100012). We acknowledge A/Prof Marijka Batterham for her assistance in the statistical analysis.

\section{REFERENCES}

R. S. Johansson and J. R. Flanagan, "Coding and use of tactile signals from the fingertips in object manipulation tasks," Nat Rev Neurosci, vol. 10, pp. 345-59, May 2009.

D. J. Atkins, D. C. Y. Heard, and W. H. Donovan, "Epidemiologic Overview of Individuals with Upper-Limb Loss and Their Reported Research Priorities," JPO: Journal of Prosthetics and Orthotics, vol. 8, pp. 2-11, 1996.

E. A. Biddiss and T. T. Chau, "Upper limb prosthesis use and abandonment: A survey of the last 25 years," Prosthetics and Orthotics International, vol. 31, pp. 236-257, 2007. E. Biddiss and T. Chau, "Upper-limb prosthetics: critical factors in device abandonment," American journal of physical medicine \& rehabilitation, vol. 86, pp. 977-987, 2007. 
[5] H. H. Ehrsson, B. Rosen, A. Stockselius, C. Ragno, P. Kohler, and G. Lundborg, "Upper limb amputees can be induced to experience a rubber hand as their own," Brain, vol. 131, pp. 3443-52, Dec 2008.

[6] M. D. Alonzo, F. Clemente, and C. Cipriani, "Vibrotactile Stimulation Promotes Embodiment of an Alien Hand in Amputees With Phantom Sensations," IEEE Transactions on Neural Systems and Rehabilitation Engineering, vol. 23, pp. 450457, 2015.

[7] L. Schmalzl, A. Kalckert, C. Ragno, and H. H. Ehrsson, "Neural correlates of the rubber hand illusion in amputees: a report of two cases," Neurocase, vol. 20, pp. 407-20, Aug 2014.

[8] B. Peerdeman, D. Boere, H. Witteveen, H. Hermens, S. Stramigioli, J. Rietman, et al., "Myoelectric forearm prostheses: State of the art from a user-centered perspective," Journal of Rehabilitation Research and Development, vol. 48, pp. 719-738, 2011.

[9] A. Ninu, S. Dosen, S. Muceli, F. Rattay, H. Dietl, and D. Farina, "Closed-Loop Control of Grasping With a Myoelectric Hand Prosthesis: Which Are the Relevant Feedback Variables for Force Control?," IEEE Transactions on Neural Systems and Rehabilitation Engineering, vol. 22, pp. 10411052, 2014.

[10] M. A. Schweisfurth, M. Markovic, S. Dosen, F. Teich, B. Graimann, and D. Farina, "Electrotactile EMG feedback improves the control of prosthesis grasping force," J Neural Eng, vol. 13, p. 056010, Oct 2016.

[11] B. Stephens-Fripp, G. Alici, and R. Mutlu, "A Review of Non-Invasive Sensory Feedback Methods for Transradial Prosthetic Hands," IEEE Access, 2018.

[12] C. Antfolk, M. D'Alonzo, M. Controzzi, G. Lundborg, B. Rosen, F. Sebelius, et al., "Artificial redirection of sensation from prosthetic fingers to the phantom hand map on transradial amputees: vibrotactile versus mechanotactile sensory feedback," IEEE Trans Neural Syst Rehabil Eng, vol. 21, pp. 112-20, Jan 2013.

[13] T. Morita, T. Kikuchi, and C. Ishii, "Development of Sensory Feedback Device for Myoelectric Prosthetic Hand to Provide Hardness of Objects to Users," Journal of robotics and mechatronics, vol. s28 no. 3, 2016.

[14] S. B. Godfrey, M. Bianchi, A. Bicchi, and M. Santello, "Influence of force feedback on grasp force modulation in prosthetic applications: A preliminary study," in 2016 38th Annual International Conference of the IEEE Engineering in Medicine and Biology Society (EMBC), 2016, pp. 5439-5442.

[15] S. Casini, M. Morvidoni, M. Bianchi, M. Catalano, G. Grioli, and A. Bicchi, "Design and realization of the CUFF - clenching upper-limb force feedback wearable device for distributed mechano-tactile stimulation of normal and tangential skin forces," in 2015 IEEE/RSJ International Conference on Intelligent Robots and Systems (IROS), 2015, pp. 1186-1193.

[16] E. Battaglia, J. P. Clark, M. Bianchi, M. G. Catalano, A. Bicchi, and M. K. O'Malley, "The Rice Haptic Rocker: skin stretch haptic feedback with the Pisa/IIT SoftHand," in World Haptics Conference (WHC), 2017 IEEE, 2017, pp. 7-12.

[17] C. Antfolk, S.-O. Bjorkman A Fau - Frank, F. Frank So Fau - Sebelius, G. Sebelius F Fau Lundborg, B. Lundborg G Fau - Rosen, and B. Rosen, "Sensory feedback from a prosthetic hand based on air-mediated pressure from the hand to the forearm skin," Journal of Rehabilitation Medicine, pp. 702-707, 2012.

[18] A. Akhtar, M. Nguyen, L. Wan, B. Boyce, P. Slade, and T. Bretl, "Passive mechanical skin stretch for multiple degree-of-freedom proprioception in a hand prosthesis," in International Conference on Human Haptic Sensing and Touch Enabled Computer Applications, 2014, pp. 120-128.

[19] C. Antfolk, C. Cipriani, M. C. Carrozza, C. Balkenius, A. Björkman, G. Lundborg, et al., "Transfer of tactile input from an artificial hand to the forearm: experiments in amputees and ablebodied volunteers," Disability and Rehabilitation: Assistive Technology, vol. 8, pp. 249-254, 2013/05/01 2013.

[20] C. Antfolk, V. Kopta, J. Farserotu, J. D. Decotignie, and C. Enz, "The WiseSkin artificial skin for tactile prosthetics: A power budget investigation," in 2014 8th International Symposium on Medical Information and Communication Technology (ISMICT), 2014, pp. 1-4.

[21] J. Gonzalez, H. Suzuki, N. Natsumi, M. Sekine, and W. Yu, "Auditory display as a prosthetic hand sensory feedback for reaching and grasping tasks," in 2012 Annual International Conference of the IEEE Engineering in Medicine and Biology Society, 2012, pp. 1789-1792.

[22] M. Vergara, J. L. Sancho-Bru, V. Gracia-Ibanez, and A. Perez-Gonzalez, "An introductory study of common grasps used by adults during performance of activities of daily living," 20140725 DCOM20150511.

[23] M. A. F. Ismail and S. Shimada, "'Robot' Hand Illusion under Delayed Visual Feedback: Relationship between the Senses of Ownership and Agency," PLOS ONE, vol. 11, p. e0159619, 2016.

[24] S. Shimada, K. Fukuda, and K. Hiraki, "Rubber Hand Illusion under Delayed Visual Feedback," PLOS ONE, vol. 4, p. e6185, 2009.

[25] T. A. Kuiken, G. Li, B. A. Lock, R. D. Lipschutz, L. A. Miller, K. A. Stubblefield, et al., "Targeted Muscle Reinnervation for Real-Time Myoelectric Control of Multifunction Artificial Arms," JAMA : the journal of the American Medical Association, vol. 301, pp. 619-628, 2009. 